Dear Author,

Please, note that changes made to the HTML content will be added to the article before publication, but are not reflected in this PDF.

Note also that this file should not be used for submitting corrections. 


\section{AUTHOR QUERY FORM}

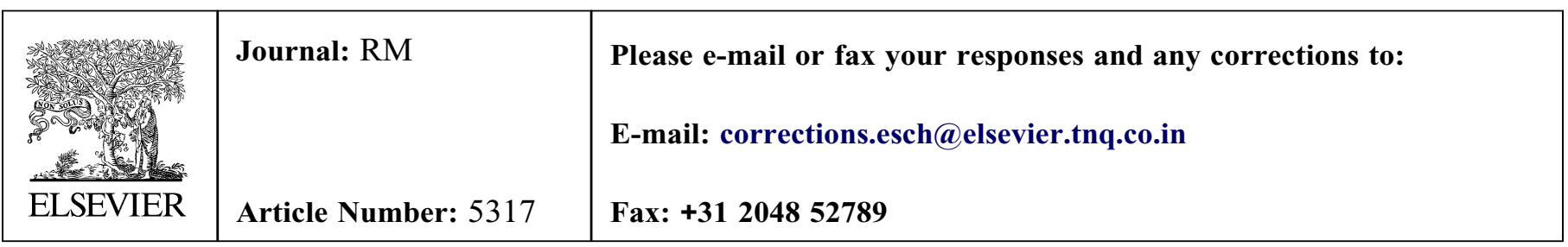

Dear Author,

Please check your proof carefully and mark all corrections at the appropriate place in the proof (e.g., by using on-screen annotation in the PDF file) or compile them in a separate list. Note: if you opt to annotate the file with software other than Adobe Reader then please also highlight the appropriate place in the PDF file. To ensure fast publication of your paper please return your corrections within 48 hours.

For correction or revision of any artwork, please consult http://www.elsevier.com/artworkinstructions.

Any queries or remarks that have arisen during the processing of your manuscript are listed below and highlighted by flags in the proof.

\begin{tabular}{|c|l|}
\hline $\begin{array}{c}\text { Location } \\
\text { in article }\end{array}$ & \multicolumn{1}{c}{$\begin{array}{c}\text { Query / Remark: Click on the Q link to find the query's location in text } \\
\text { Please insert your reply or correction at the corresponding line in the proof }\end{array}$} \\
\hline Q1 & $\begin{array}{l}\text { Please check the sentence 'Such results suggest that the supposedly interference...' for clarity, and correct } \\
\text { if necessary. } \\
\text { Q2 }\end{array}$ \\
Q3 & $\begin{array}{l}\text { Extra closing parentheses have been deleted in Table } 2 \text { equations. Please check, and correct if necessary. } \\
\text { Uncited references: This section comprises references that occur in the reference list but not in the body of } \\
\text { retained in this section. } \\
\text { Please confirm that given names and surnames have been identified correctly. } \\
\text { Please check this box or indicate } \\
\text { your approval if you have no } \\
\text { corrections to make to the PDF file }\end{array}$ \\
\hline
\end{tabular}

Thank you for your assistance. 


\title{
ESR dosimetry of optically bleached quartz grains extracted from Plio- Quaternary sediment: Evaluating some key aspects of the ESR signals associated to the Ti-centers
}

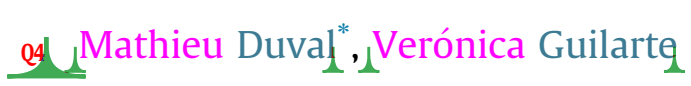 \\ Centro nacional de investigación sobre la evolución humana (CENIEH), Paseo de Atapuerca s/n, 09002 Burgos, Spain
}

\section{H I G H L I G H T S}

- We study some key aspects of ESR dosimetry of quartz grains based on the Ti-center.

- We explore several options to evaluate the ESR intensity.

- The ESR-Ti signal shows a systematic non-monotonic behavior with the absorbed dose.

- The classic SSE function can simply not describe the "radiation bleaching" phenomenon.

- The use of another fitting function is recommended.

\section{A R T I C L E I N F O}

\section{Article history:}

Received 25 November 2013

Received in revised form

23 August 2014

Accepted 25 October 2014

Available online $\mathrm{xxx}$

\section{Keywords:}

Electron spin resonance (ESR) dating

Optically bleached quartz grains

Ti center

Geochronology

\begin{abstract}
A B S T R A C T
The present study aims at investigating several key aspects of ESR dose reconstruction of quartz grains based on the analysis of the Ti-center: (i) the evaluation of the ESR intensity, (ii) its impact on measurements precision, and (iii) the potential of various fitting functions to describe the behavior of the signal with the radiation absorbed dose.

In contrast with the Al center, the various Ti centers have quite low ESR signal intensities. It is thus crucial to adapt the experimental conditions for the optimization of the signal-to-noise ratio and to perform repeated measurements in order to take into consideration the uncertainty associated to angular dependence of the signal as well as day-to-day variations. Several options (named A to E) for evaluating the ESR intensity of the $\mathrm{Ti}-\mathrm{Li}$ and $\mathrm{Ti}-\mathrm{H}$ centers are explored and some of them yield too much experimental uncertainty (e.g. options $C$ and E) and are apparently not suitable for accurate ESR dosimetry. In contrast, options $\mathrm{A}$ and $\mathrm{D}$ usually provide similar equivalent dose $\left(D_{\mathrm{E}}\right)$ results and can be used together for assessing the dose absorbed by the Ti-Li center.

Our results show a systematic non-monotonic behavior of the ESR signal of the Ti center with the dose, which raise some questions about the suitability of the single saturating exponential (SSE) function that is classically used in ESR dating. Therefore we explored the potential of other functions that can describe the "radiation bleaching" phenomenon observed at high doses. We recommend the use of a specific fitting function (called Ti-2 in the present paper) previously proposed by Woda and Wagner (2007) for any dose reconstruction, and define some criteria to ensure a good fitting. The SSE function provides $D_{\mathrm{E}}$ results that are, in most cases, relatively consistent with those derived from the Ti-2, suggesting that the Ti centers apparently follow a SSE behavior up to, at least, 6.0-6.5 kGy However, the reliability of the $D_{\mathrm{E}}$ values obtained with the SSE seems quite dependent on the accuracy of the ESR intensities of the increasing domain of the dose response curve. We also recommend the definition of criteria to check the reliability of the $D_{\mathrm{E}}$ results.
\end{abstract}

(c) 2014 Elsevier Ltd. All rights reserved.

\section{Introduction}

Because quartz is an ubiquitous mineral that may be found in

* Corresponding author. Tel.: +34947 040800; fax: +34947040810.

E-mail address: mathieu.duval@cenieh.es (M. Duval). almost any Quaternary sedimentary context, its use as a geochronometer to date sediment deposition is of special interest for 
archaeological or geological purpose. Electron Spin Resonance (ESR) dating of optically bleached quartz grains is based on a similar principle to Optically Stimulated Luminescence (OSL) dating: the resetting of a signal under sunlight exposure. Three different paramagnetic centers are commonly used, the Germanium (Ge) center, the Aluminum ( $\mathrm{Al}$ ) center and the Titanium ( $\mathrm{Ti}$ ) center. If the first one can be measured at room temperature, it is usually hardly present in natural samples, which raise some questions about the reliability of the ESR age estimates that may be obtained (e.g. Walther and Zilles, 1994; Zhao et al., 2012). In contrast, Al and Ticenters are usually present in most of the Quaternary quartz grains but may only be properly resolved at low temperature $(<\sim 120 \mathrm{~K})$. Each center shows different characteristics in terms of thermal stability, optical bleaching kinetics or radiation sensitivity, which lead some authors to propose the combined study of both centers (sometimes called "multiple center method") to evaluate the reliability of the equivalent dose $\left(D_{\mathrm{E}}\right)$ values that are obtained (e.g. Toyoda et al., 2000).

However, the amount of information available may significantly vary from one center to another. So far, the Al center is the most studied paramagnetic center for geochronological purpose: its behavior with the absorbed dose as well as its bleaching kinetics are now quite well-known (e.g. Duval, 2012; Voinchet et al., 2003), and its main specificity lies in the presence of an unbleachable residual ESR intensity that has to be accurately considered in the evaluation of the past radiation dose absorbed by the sample (e.g. Tissoux et al., 2012). In comparison with the Al center, the Ti center may potentially offer two advantages in geochronology: (i) its ESR intensity may be fully reset to zero by optical bleaching (i.e. no residual ESR intensity), (ii) its bleaching kinetics is significantly much faster than that of the Al center (Toyoda et al., 2000), and therefore there is less uncertainty about the complete bleaching of the signal prior to burial. However, the exact potential of this center for dating is still somewhat unclear, since several basic points need be further investigated.

For example, there is no information available in the literature about the repeatability of the ESR measurements or the precision of the ESR intensities, whereas this is potentially a major source of uncertainty in the evaluation of the equivalent dose $\left(D_{\mathrm{E}}\right)$. In addition, the way of measuring the ESR intensity of the Ti-center may vary from one author to the other (e.g. Tissoux et al., 2008; Liu et al., 2010; Shimada et al., 2013; Rink et al., 2007), and so far only one comparison study has been partially focused on this aspect, but based on a limited number and diversity of samples (Tissoux et al., 2008). Finally, the behavior of the ESR signal associated to the Ti center with the absorbed dose has been little studied. To our knowledge, the ESR dating applications dealing with this center are almost exclusively based on the use of a single saturating exponential (SSE) function for the evaluation of the $D_{\mathrm{E}}$ (e.g. Rink et al., 2007; Burdette et al., 2012; Liu et al., 2010; Tissoux et al., 2008), with very rare exceptions (e.g. Liu et al., 2013). However, the choice of a given fitting function is sometimes obviously not supported by the ESR data points and the reliability of the $D_{\mathrm{E}}$ values that were calculated may be thus reasonably questioned (e.g. Tissoux et al., 2007), even if final ESR ages are apparently consistent with results derived from another independent method (e.g, Liu et al., 2013). In fact, Woda and Wagner (2007) showed that the Ti center has a specific non-monotonic evolution with the absorbed dose, unlike the $\mathrm{CO}_{2}^{-}$radicals in enamel (Duval et al., 2009) or the Al center in quartz (Duval, 2012). In other words, the ESR intensity is known to decrease at high dose values and this specific behavior, sometimes called "radiation bleaching" (Euler and Kahan, 1987) or "radiation damage" (Lawless et al., 2005), can simply not be described by a SSE function. Nevertheless, so far this has been never taken into consideration when using the Ti-center for ESR dating purpose.

The present study aims at investigating several key aspects of ESR dose reconstruction of quartz grains based on the Ti-center: (i) the evaluation of the ESR intensity, (ii) its impact on measurements precision, and (iii) the potential of various fitting functions, other than the SSE, to describe the behavior of the signal with the radiation absorbed dose.

\section{Brief description of the Ti center}

$\mathrm{Ti}^{4+}$ is one of the cations that may substitute the silicon atoms in the crystalline network of quartz, creating thus a defect usually written as $\left[\mathrm{TiO}_{4}\right]^{0}$ (Weil, 1984) and precursor of the so-called Ti center. Natural irradiation of the quartz induces some electron movements in the crystalline network, and $\mathrm{Ti}^{4+}$ may trap an electron. In that case, the charge excess may be compensated by a cation, usually called $\mathrm{M}^{+}$, incorporated in an interstitial position. The paramagnetic center is thus usually defined as $\left[\mathrm{TiO}_{4} / \mathrm{M}^{+}\right]^{0}$, with $\mathrm{M}^{+}$being either $\mathrm{Li}^{+}, \mathrm{Na}^{+}$or $\mathrm{H}^{+}$(see further details in Duttine, 2005; Götze et al., 2001). Depending on the compensating cation, there are three kinds of Ti centers, which may be called $\mathrm{Ti}-\mathrm{Li}, \mathrm{Ti}-\mathrm{Na}$ or Ti-H center.

Each center has a specific ESR signal: the Ti-H center is defined by three main absorption lines at $g_{1}=1.986, g_{2}=1.931, g_{3}=1.915$, where each one is split into a doublet due to the proton; $\mathrm{Ti}-\mathrm{Li}$ is defined by a $g$ factor with three different components: $g_{1}=1.979$, $g_{2}=1.931, g_{3}=1.913$, as well as Ti-Na, with $g_{1}=1.968, g_{2}=1.954$, $g_{3}=1.899$ (Okada et al., 1971; Rinnenberg and Weil, 1972; Toyoda et al., 2000). An example of ESR signal is given in Fig. 1.

\section{Material and methods}

In order to get a good overview of the question, we selected six very diverse Plio-Pleistocene sedimentary quartz samples from the Mediterranean area, with distinct geological contexts and origins. Details may be found in Table 1.

Sediment samples were prepared in the laboratory under red light (dark room conditions). The 100-200 $\mu \mathrm{m}$ size fraction was collected after wet sieving. $\mathrm{HCl}(36 \%)$ was used to dissolve carbonates and $\mathrm{H}_{2} \mathrm{O}_{2}(30 \%)$ to eliminate organic matter. Heavy minerals and feldspars were removed with Sodium Polytungstate solutions at $d=2.72$ and $d=2.62 \mathrm{~g} / \mathrm{ml}$, respectively. Then, magnetic minerals were eliminated using neodymiun magnets. The resulting samples were treated with $\mathrm{HF}(40 \%)$ for 40 min to eliminate the remaining feldspars and to etch quartz grains. Finally, $\mathrm{HCl}$ (18\%) was added in order to remove any soluble fluoride. A final sieving was performed to remove grains $<80 \mu \mathrm{m}$ size. Each quartz sample was then divided into 12 to 14 aliquots that were irradiated with a calibrated Gammacell-1000 Cs-137 gamma source (dose rate $=7.3 \mathrm{~Gy} / \mathrm{min}$ ) up to $25-60 \mathrm{kGy}$, depending on the sample. ESR measurements were carried out at CENIEH (Burgos, Spain), with an EMXmicro 6/1Bruker X-band ESR spectrometer coupled to a standard rectangular ER 4102ST cavity. To ensure constant experimental conditions over time, the temperature of the water circulating in the magnet is controlled and stabilized at $18{ }^{\circ} \mathrm{C}$ by a water-cooled Thermo Scientific NESLAB ThermoFlex 3500 chiller, and the temperature of the room is kept constant at $20^{\circ} \mathrm{C}$ by an air conditioning unit. ESR measurements were performed at low temperature ( $90 \mathrm{~K})$ using a ER4141VT Digital Temperature control system based on liquid nitrogen cooling. Further details about the setup and about its stability over time can be found in Duval and Guilarte Moreno (2012).

Given the low intensity of the ESR signal usually measured for the $\mathrm{Ti}$ center, experimental conditions of ESR measurements 
A
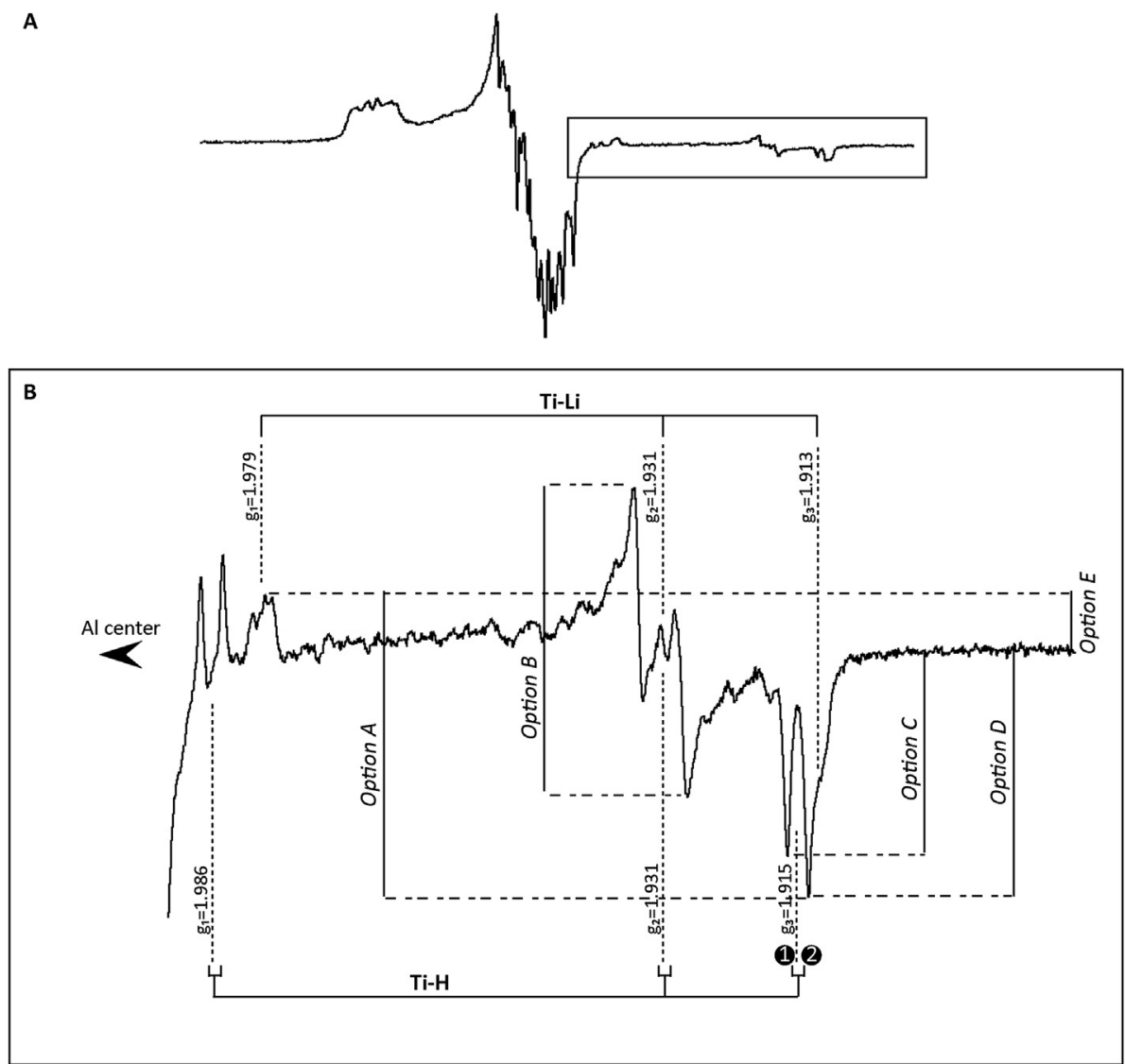

Fig. 1. Example of ESR signals obtained at low temperature ( $\sim 90 \mathrm{~K})$ from natural quartz samples. (A): ESR spectrum showing both Al and Ti center signals. (B): Zoom on the ESR signal of the Ti centers. In that case, the Ti-Na center is not observed. The various options for the evaluation of the ESR intensity (A-E) are indicated.

routinely used for the Al center can simply not be used and have to be specifically optimized to improve the signal-to-noise $(S / N)$ ratio. In that regard, the following ESR acquisition parameters were used: 1 to 10 scans (depending on the signal intensity), $5 \mathrm{~mW}$ microwave power, 1024 points resolution, $20 \mathrm{mT}$ sweep width, $100 \mathrm{kHz}$ modulation frequency, $0.1 \mathrm{mT}$ modulation amplitude, $60 \mathrm{~ms}$ conversion time and $10 \mathrm{~ms}$ time constant. For a given sample, each aliquot was measured up to three times after a $\sim 120^{\circ}$ rotation in the cavity, in order to take into account the angular dependence of the ESR signal. This results in a total measurement time of 2.5-3 h for all the aliquots of a given sample, ensuring thus stable experimental conditions for repeatable measurements (Duval and Guilarte Moreno, 2012). This procedure was repeated over three to four different days without removing the quartz samples from the ESR tubes between measurements. Depending on the sample mass available, aliquots of between 138 and $303 \mathrm{mg}$ were inserted in the tubes. For a given sample, the mass variation between all the aliquots was between 0.1 and $0.2 \mathrm{mg}$, i.e. $<0.1 \%$ of the mass, ensuring thus similar resonance conditions in the ESR resonator.

We studied five ways of evaluating the intensity of the ESR signal of the Ti-center (see Fig. 1):

- Option A: Peak-to-peak amplitude measurement between $g=1.979$ and the bottom of the peak around $g=1.913-1.915$ (peak labeled "2"), without baseline correction (e.g. Rink et al., 2007; Liu et al., 2010).

- Option B: Peak-to-peak amplitude measurement around $g=1.931$.
- Option C: Peak-to-baseline amplitude measurement at $g=1.915$ (peak labeled "1" in Fig. 1) without baseline correction (e.g. Tissoux et al., 2008).

- Option D: Peak-to-baseline amplitude measurement around $g=1.913-1.915$ (peak labeled "2" in Fig. 1) without baseline correction (e.g. Tissoux et al., 2008).

- Option E: Peak-to-baseline amplitude measurement at $g=1.979$ without baseline correction.

Intensities were extracted from the ESR spectrum of each aliquot, and then corrected by the corresponding receiver gain value, number of scans, aliquot mass and a temperature correction factor (Duval and Guilarte Moreno, 2012).

Fitted parameter values (including $D_{\mathrm{E}}$ ) were derived from fitting procedures carried out with the Microcal OriginPro8.5 software using a Levenberg-Marquardt algorithm by chi-square minimization. Errors of the fitted parameter errors are assessed through the square root of the diagonals covariance matrix values (see the Origin 8 User Guide for further details). Further details about the potential of this algorithm for non linear fitting may be found in Hayes et al. (1998). Data points were not weighted, given the nonmonotonic behavior of the ESR signal. Indeed, if data weighting by the inverse of the squared ESR intensity $\left(1 / I^{2}\right)$ is classically recommended for ESR dose reconstruction (e.g. Grün and Brumby, 1994 ) in order to reduce the leverage of high dose points (Lyons et al., 1992), in this case it might produce the opposite effect due to the low ESR intensities of the most irradiated points. The goodness-of-fit was assessed through the adjusted $r$-square $\left(r^{2}\right)$ value, which accounts for the degrees of freedom of the system, 
Table 1

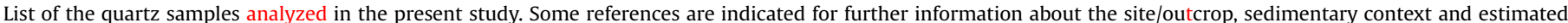
chronology.

\begin{tabular}{|c|c|c|c|c|}
\hline Sample & Site/outcrop & Estimated chronology & Sedimentary context & Reference \\
\hline BUR1121 & Tejares (Spain) & Plio-Pleistocene (>2 Ma) & Alluvial deposits ("Rañas”) & Lemartinel (1997) \\
\hline LEZ1001 & Lézignan-la-Cèbe (France) & Early Pleistocene ( 1.6 Ma) & Alluvial deposits & Crochet et al. (2009) \\
\hline TE1002 & Sima del Elefante (Spain) & Early Pleistocene ( 1.2 Ma) & Karst infilling & Carbonell et al. (2008) \\
\hline FN1001 & Fuente Nueva-3 (Spain) & Early Pleistocene $(\sim 1.2 \mathrm{Ma})$ & Fluvio-lacustrine deposits & Martínez-Navarro et al. (1997), Oms et al. (2011) \\
\hline AB1104 & Aïn Boucherit (Algeria & Early Pleistocene $(\sim 1.8 \mathrm{Ma})$ & Alluvial deposits & Sahnouni et al. $(1998,2011)$ \\
\hline PN11-3 & Pirro Nord (Italy) & Plio-Pleistocene (1.4 Ma) & Karst infilling & Arzarello et al. (2012); Pavia et al. (2012) \\
\hline
\end{tabular}

contrary to the classical coefficient of determination $r^{2}$ (see the Origin 8 User Guide for further details).

Five fitting functions were tested (see Table 2):

- Ti-1: This is the SSE function that is almost exclusively employed for ESR dating applications based on the Ti center (e.g. Rink et al., 2007; Burdette et al., 2012; Liu et al., 2010; Tissoux et al., 2008). The use of this function suggests that the $\mathrm{Ti}$ signal is dominated by a single component that saturates at high doses.

- Ti-2: This empirical function was proposed by Woda and Wagner (2007) to describe the decreasing behavior of the ESR intensities at high doses.

- Ti-3: This empirical function was also proposed by Woda and Wagner (2007) as an alternative to function Ti-2, to describe an exponential decay for doses up to $40 \mathrm{kGy}$.

- Ti-4: This function was interpreted by Charalambous and Petridou (1976) as describing a competition between the creation and the destruction of paramagnetic centers, the first process dominating at relatively low dose range and the latter at high dose range. However, these authors do not provide real physical explanation for this model, for which Woda and Wagner (2007) considered it as an empirical function. Ti-4 may be considered as an equivalent to the double saturating exponential function (Duval et al., 2009), except that the second term is negative in this case.

- Ti-5: This is a simplified version of $\mathrm{Ti}-4$, where the second exponential negative term is approximated to a linear term, similarly to the exponential + linear function (Duval, 2012).

Contrary to function Ti-1 (SSE), the four other functions consider that the ESR signal is dominated by more than one component and can actually describe the radiation bleaching process at high doses.

\section{Results and discussion}

\subsection{Composition of the Ti ESR signal}

Fig. 2 shows an example of the diversity of ESR signals of the Ti centers that could be observed in natural quartz samples. All the spectra have been normalized in gain, number of scan, temperature and mass and are shown at the same X- and Y-scales. Basically, the existence of at least three different Ti centers may generate a composite ESR signal whose shape may strongly depend on the proportion of each kind of Ti center present in the quartz sample. Among the three centers, the $\mathrm{Ti}-\mathrm{Na}$ is not systematically observed in every sample (e.g. Toyoda et al., 2000). Actually, none of the samples shown in Fig. 2 have this specific ESR signal. The Ti-Li signal is systematically present in all samples, unlike the $\mathrm{Ti}-\mathrm{H}$ which may be sometimes almost absent. This is illustrated by Fig. 2 where all ESR spectra are roughly classified according to their apparent relative proportion of $\mathrm{Ti}-\mathrm{H}$ centers vs $\mathrm{Ti}-\mathrm{Li}$ ones, from very low (top) to high proportion (bottom). The ESR intensity of the Ti signals may also significantly vary from one site to another, regardless the total dose absorbed by the samples. This variability in signal intensity and composition may be directly related to the chemical composition (and trace element contents) of the quartz grains, which is especially useful for sediment characterization and provenance studies (e.g. Duttine et al., 2002; Shimada et al., 2013).

When extracting an intensity value from an ESR signal, it is important to have a precise idea of the composition of the signal and try to remove, or at least minimize, the influence of interfering signals. In the case of the Ti centers, it is important to make sure that the $\mathrm{Al}$ signal is not interfering too much in the Ti signal. Indeed, the Al signal may be $>10$ times stronger than the Ti signal and the last peak is quite close from the peaks at $g=1.986$ and also, perhaps, $g=1.979$ of the $\mathrm{Ti}-\mathrm{H}$ and $\mathrm{Ti}-\mathrm{Li}$ centers, respectively (Fig. 1). Therefore, the use of these peaks (especially the peak at $g=1.986$ ) for the evaluation of the ESR intensity of the Ti center may be reasonably questioned. To remove the influence of the $\mathrm{Al}$ signal, a baseline correction prior to data extraction may be envisaged but at least two field domains with only background (i.e. without any signal) have to be selected, preferentially before and after the studied ESR signal. In that case, there are not so many solutions, given that the field range located between the peaks at $g=1.979$ and $g=1.931$ (roughly from $\sim 3440$ to $\sim 3500$ G, Fig. 2) is probably not a proper background area. In the field range $>3560 \mathrm{G}$ (Fig. 2) no ESR signal is observed, and may thus be suitable as a background domain. However, the other selected domain would have to be before the Al signal in terms of magnetic field values. Basically, this means that the Al signal would have to be systematically measured together with the Ti one in order to get a proper background correction (Fig. 1 A). This option would be especially unpractical, considering the difference in signal intensities of both centers ( $\mathrm{Al}$ and $\mathrm{Ti}$ ). In fact, an appropriate receiver gain level for the Ti center would cause saturation of the Al signal, whereas a proper

Table 2

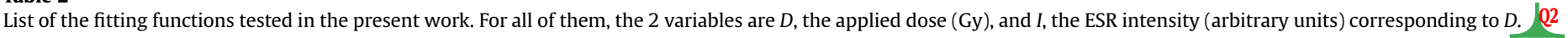

r

\begin{tabular}{|c|c|c|}
\hline Function & Equation & Fitted param. \\
\hline Ti-1(SSE) & $I(D)=I_{\mathrm{sat}} *\left(1-e^{-\left(D+D_{E}\right) / D_{0}}\right)$ & (3): $I_{\mathrm{sat}}, D_{0}, D_{\mathrm{E}}$. \\
\hline $\mathrm{Ti}-2$ & $I(D)=a^{*}\left(e^{-\left(D+D_{E}\right) / D_{1}}\right)-e^{-\left(D+D_{E}\right) / D_{2}}$ & (4): $a, D_{1}, D_{2}, D_{\mathrm{E}}$ \\
\hline Ti-3 & $I(D)=a_{1} * e^{-\left(D+D_{E}\right) / D_{1}}+a_{2} * e^{-\left(D+D_{E}\right) / D_{2}}-\left(a_{1}+a 2\right) * e^{-\left(D+D_{E}\right) / D_{3}}$ & (6): $a_{1}, a_{2}, D_{1}, D_{2}, D_{3}, D_{\mathrm{E}}$. \\
\hline $\mathrm{Ti}-4$ & $I(D)=a_{1} *\left(1-e^{-\left(D+D_{E}\right) / D_{1}}\right)-a_{2} *\left(1-e^{-\left(D+D_{E}\right) / D_{2}}\right)$ & (5): $a_{1}, a_{2}, D_{1}, D_{2}, D_{\mathrm{E}}$ \\
\hline Ti-5 & $I(D)=a_{1} *\left(1-e^{-\left(D+D_{E}\right) / D_{1}}\right)-a_{2} *\left(D+D_{E}\right)$ & (4): $a_{1}, a_{2}, D_{1}, D_{\mathrm{E}}$ \\
\hline
\end{tabular}



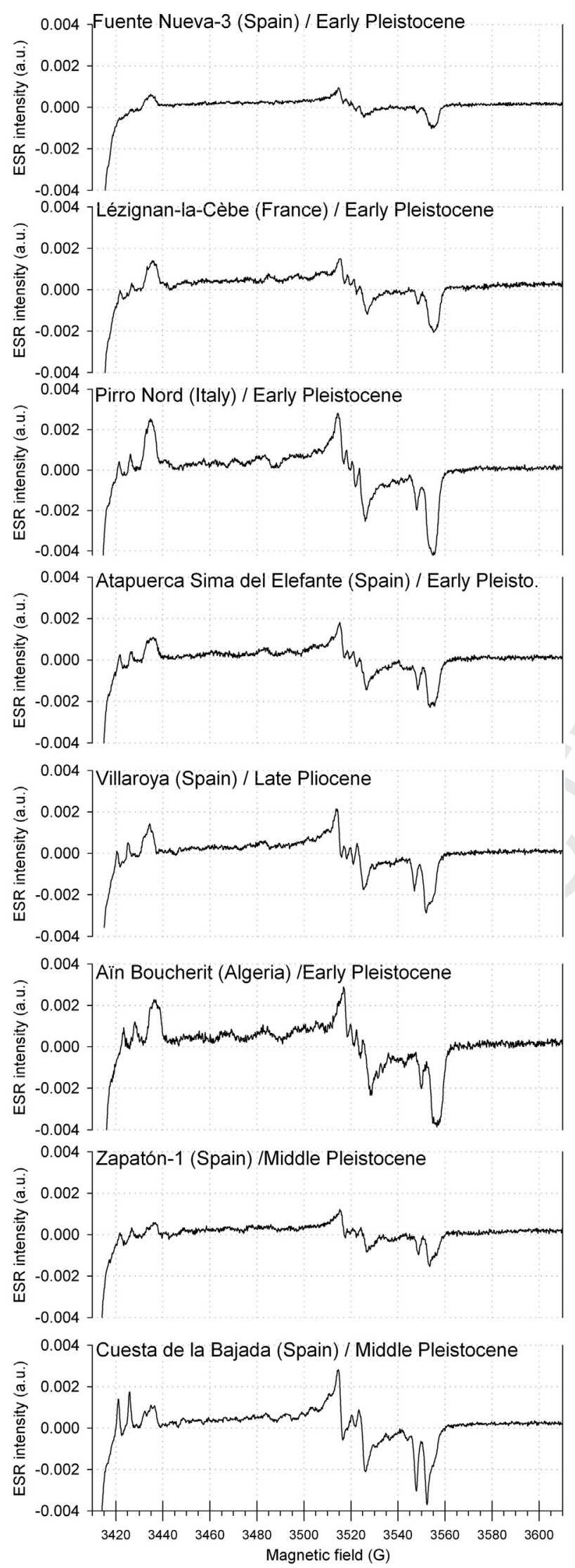

Fig. 2. Examples of Ti-ESR signals that may be encountered in natural quartz sediment samples from various sites located in the Mediterranean area. ESR spectra are roughly classified according to the proportion of the $\mathrm{Ti}-\mathrm{H}$ centers relatively to the $\mathrm{Ti}-\mathrm{Li}$ gain for the $\mathrm{Al}$ center will result in a very weak Ti signal, being required to increase the number of scans. This will eventually make the measurement time too long given the sweep width $(\sim 400 \mathrm{G})$ to ensure a good stability of the experimental conditions throughout the successive measurements of all the aliquots of a given sample. Therefore, we do not recommended to use the peak at $g=1.986$ for the evaluation of the ESR intensity.

The peak at $g=1.979$ is presumably only made by $\mathrm{Ti}-\mathrm{Li}$, whereas the peaks around $g=1.931$ are composed by a mixture of $\mathrm{Ti}-\mathrm{Li}$ and $\mathrm{Ti}-\mathrm{H}$. The left peak at $\mathrm{g}=1.915$ (labeled 1, Fig. 1 ) is very likely dominated by $\mathrm{Ti}-\mathrm{H}$, but an influence of $\mathrm{Ti}-\mathrm{Li}$ might not be neglected given the proximity of the peak at $g=1.913$. In contrast the right peak around $g=1.915$ (labeled 2 in Fig. 1 ) is influenced by the $\mathrm{Ti}-\mathrm{Li}$ at $g=1.913$ but each peak can hardly be isolated in Xband ESR spectrometry. For some of the samples, a slope inflexion may be sometimes observed on the right side of the peak, but not systematically (Fig. 2), which results very complicated to measure the peak at $g=1.915$ in the way suggested by Toyoda et al. (2000). In that regard, some specific ESR measurements should be performed in the future with a modulation amplitude lower than $0.1 \mathrm{mT}$ to see whether this peak shoulder can be better resolved and, may be, isolated. However, so far the only solution for the moment lies in measuring the maximum ESR intensity of the peak around $g=1.915-1.913$, knowing that this might be a mixture of contributions from $\mathrm{Ti}-\mathrm{Li}$ and $\mathrm{Ti}-\mathrm{H}$ centers.

Consequently, it seems that the peaks around $g=1.931$, $g=1.915, g=1.913$ and $g=1.979$ would be the best options to evaluate the ESR intensity of the Ti center. Five options are thus explored (see Section 3). To resume, option B is very likely a mixture of $\mathrm{Ti}-\mathrm{Li}$ and $\mathrm{Ti}-\mathrm{H}$. Option $\mathrm{C}$ and $\mathrm{E}$ are made by a single contribution of the $\mathrm{Ti}-\mathrm{H}$ and $\mathrm{Ti}-\mathrm{Li}$ centers, respectively. In contrast, option $\mathrm{D}$ is very likely dominated by the $\mathrm{Ti}-\mathrm{Li}$ center, with nevertheless a (hardly quantifiable) contribution from the $\mathrm{Ti}-\mathrm{H}$ center, given the two absorption lines at $g=1.913$ and $g=1.915$ (labeled 2, Fig. 1). In comparison, the relative contribution of the $\mathrm{Ti}-\mathrm{H}$ center is even smaller in option A, simply because the amplitude measurement is made with the peak at $g=1.979$, rather than with the baseline. The influence of each contribution on the final $D_{\mathrm{E}}$ value will be assessed in Section 4.3.

\subsection{ESR dose response curves (DRC)}

Perhaps the most striking observation is that the Ti centers have systematically a non-monotonic behavior with the irradiation dose (Figs. 3 and 4): unlike the radiation induced ESR signal of tooth enamel that may constantly increase up to at least $50 \mathrm{kGy}$ (e.g. Duval et al., 2009), and the ESR signal of Al center that shows no saturation effects at high doses (Duval, 2012), the Ti signal reaches a maximum ESR intensity roughly around $10 \mathrm{kGy}$ and then decreases at higher doses. Similar observations were previously made by Woda and Wagner (2007) and Tissoux et al. (2007). This phenomenon called "radiation bleaching" or "radiation damage" depending on the authors (Euler and Kahan, 1987; Lawless et al., 2005; Woda and Wagner, 2007) could be induced by a competition between single and double electron capture. Woda and Wagner (2007) suggest that during the irradiation process a second electron could be captured by the charge compensating ion, resulting in the creation of a neutral $\mathrm{M}^{0}$ center and a locally uncompensated paramagnetic center $\left[\mathrm{TiO}_{4}\right]^{-}$. This center, unstable at room temperature (Okada et al., 1971), would need an alternative

centers, from low (Fuente Nueva-3 spectrum) to high (Cuesta de la Bajada spectrum). The ESR intensity of each spectrum has been corrected in gain, temperature, mass and number of scans and are all shown with the same X- and Y-scale. 


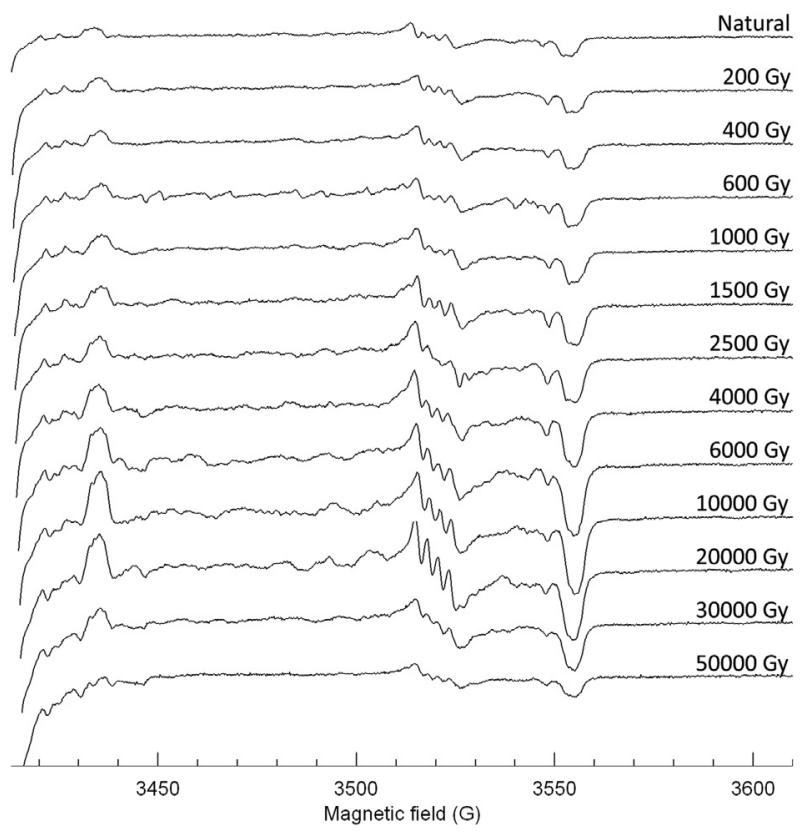

Fig. 3. Examples of ESR spectra obtained for each aliquot of sample LEZ1001.

charge compensating ion or stabilizing mechanism and consequently may not contribute to the main radiation induced ESR signal anymore.

In order to get comparable data, ESR intensities were normalized to that of the natural aliquot for each sample (Fig. 4). Options A, $\mathrm{B}, \mathrm{D}$ and $\mathrm{E}$ show a somewhat similar behavior and relative ESR intensities:

- The relative ESR intensities derived from options A, B, D and E reach a maximum value at doses somewhere between 6 and $10 \mathrm{kGy}$, depending on the sample.

- Relative ESR intensities derived from option B are on average 7\% higher than those obtained with option $\mathrm{A}$, and $31 \%$ of the data points are within $\pm 5 \%$.

- Options A and D yield quite similar relative ESR intensities. Option D has ESR data that are on average only $1 \%$ higher than those derived from option $A$ and $58 \%$ are within $\pm 5 \%$, showing thus a similar radiation sensitivity.

- Relative ESR intensities obtained with option E are on average $4 \%$ lower than those obtained with option A and $46 \%$ of the data points are within $\pm 5 \%$.

In contrast, Options $C$ has a behavior significantly different from the others options:

- For the six samples, relative ESR intensities derived from option $C$ are systematically higher than those from the other options in the increasing domain of the DRC (from $D=0$ Gy to $D \sim 4 \mathrm{kGy}$ ). This higher radiation sensitivity might make the $\mathrm{Ti}-\mathrm{H}$ center (=option C) of special interest to detect small $D_{\mathrm{E}}$ values.

- Lower irradiation doses are required to start the radiation bleaching process. For five of the samples, the ESR intensity reaches a maximum value one or several dose steps before the other options. In addition, for four of the samples (BUR1121, LEZ1001, TE1002, PN11-3), the maximum ESR intensities are significantly lower than those from the other options.

If the samples all show similar trends, some differences may be nevertheless observed. Fig. 5 shows the ESR intensities derived from Option A and illustrates the variability of the radiation sensitivity between the samples. If part of this apparent variability may be linked to the geological dose naturally absorbed by each sample, the ESR intensities may nevertheless vary by a factor up to 2.8 between AB1104 and LEZ1001 at $D \sim 10 \mathrm{kGy}$. The former sample has a value of ESR intensity lower than that of the natural aliquot at doses $>20 \mathrm{kGy}$, while the dose threshold is $>30 \mathrm{kGy}$ for samples TE1002, LEZ1001, FN1001 and PN11-3.

\subsection{Variability of the ESR intensities over repeated measurements}

For a given sample (12-14 aliquots), each aliquot was measured up to 3 times after $\sim 120^{\circ}$ of rotation in the cavity. This procedure was repeated over three to four different days. We observed that day-to-day variations of the ESR intensities can hardly be differentiated from the variability of the ESR intensities due to angular dependence of the signal. Consequently, for each aliquot a mean ESR intensity value and a standard deviation were derived from all the repeated measurements. This standard deviation may be used as a proxy to evaluate the precision of the ESR intensities obtained for the Ti center. Considering all the aliquots of a given sample, the mean relative standard deviation of the ESR intensities can be obtained for the different options to measure the Ti center (Table 3, columns 2-6). This precision is significantly lower than that usually afforded with the Al center: when considering the 5 samples (i.e. 78 aliquots), the mean relative standard deviation of the ESR intensities is of $3.4 \%$ with option A, 5.1\% with option B, $13.9 \%$ with option C, $4.0 \%$ with option D and $7.8 \%$ with option $E$ (Table 2). Fig. 6 provides an extreme example of high variability, by showing all the ESR spectra from the repeated measurement of a given aliquot from sample LEZ1001 ( $D=2500 \mathrm{~Gy}$ ). When focusing only on the points with $D>10 \mathrm{kGy}$, the mean variability of the ESR intensity is systematically higher (Table 2): $5.0 \%$ with option A, $6.2 \%$ with option $B$, $22.3 \%$ with option C, $5.4 \%$ with option D and $12.6 \%$ with option $E$.

This variability may be mostly explained by several factors. Firstly, there is a clear correlation between the $S / N$ ratio and the variability of the ESR intensity. By definition, the higher the absolute ESR intensity, the lower its variance over repeated measurements (Maghraby, 2010). Option A shows systematically the highest absolute ESR intensities, and thus the best $S / N$ ratio, followed by Option B, Option D, then options E and C (Table 4). This trend may be correlated to that of the variability of the ESR intensities over repeated measurements, which may be classified in increasing order as follows: option A, D, B, E and C. In that case, the inversion between options $D$ and $B$ may be explained by the fact that option $\mathrm{B}$ is a mix of contributions from $\mathrm{Ti}-\mathrm{Li}$ and $\mathrm{Ti}-\mathrm{H}$, and may be thus also more influenced by the high variability of $\mathrm{Ti}-\mathrm{H}$ over repeated measurements (see option C) than option D. In addition, this variability is also very likely depending on the heterogeneity of the quartz samples, which may generate a powder sample with preferential orientations, inducing an angular dependence of the ESR signal in the ESR resonator. Other sources of uncertainty may be involved, such as a difference in glassware or stability of the ESR spectrometer (Duval and Guilarte Moreno, 2012), but they have very likely minor influence on the variability of the ESR intensity in comparison with the other parameters previously mentioned.

These basic observations demonstrate the necessity of carrying out repeated measurements of the Ti-center, in order to get meaningful mean ESR intensities. However, at the same time, some options such as $C$ and $E$ show such a high variability (13.9\% and 7.8\%, respectively) that their interest for dosimetric purpose may be reasonably questioned. 

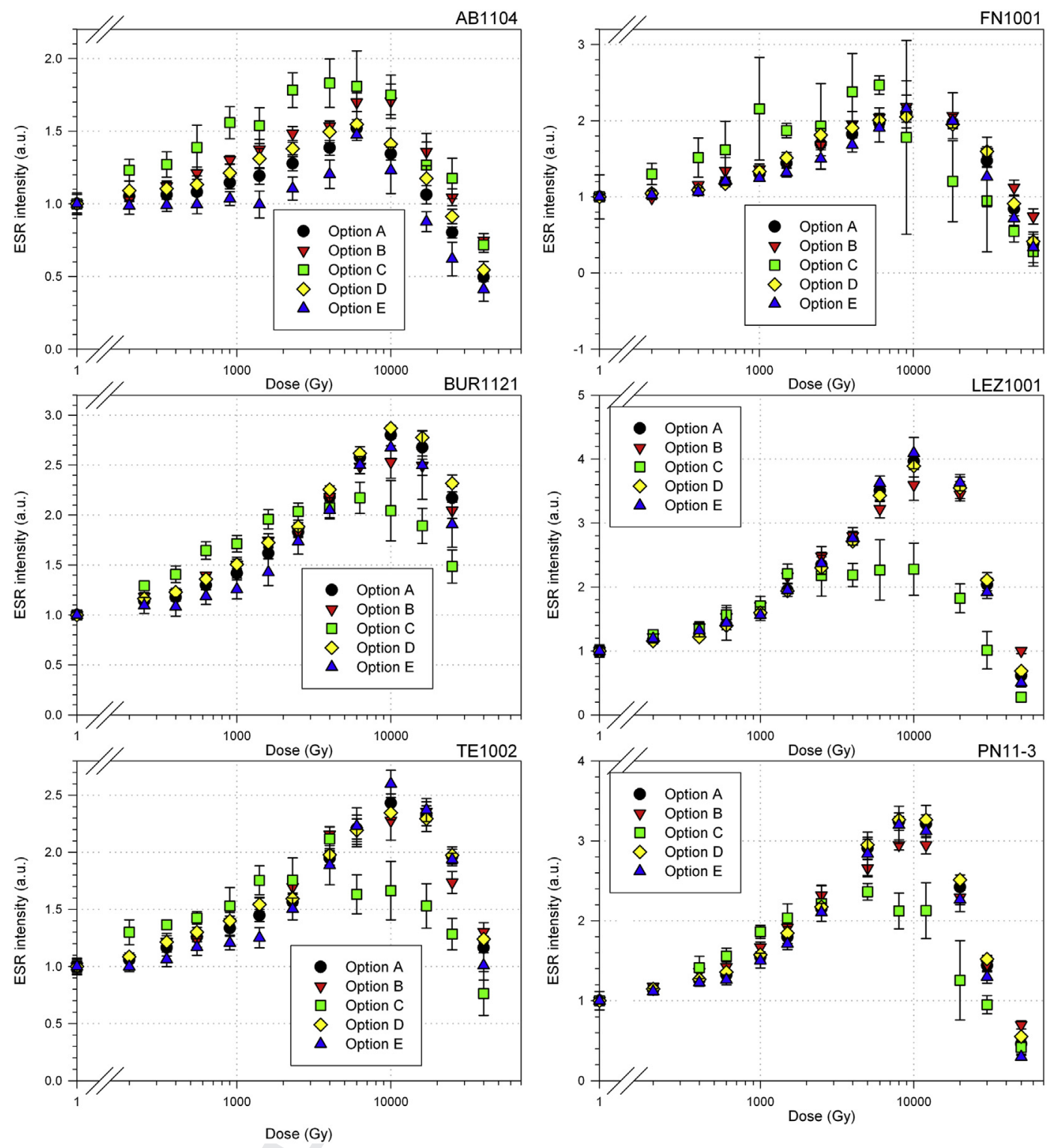

Fig. 4. ESR Dose response curves obtained for the five options of ESR intensity evaluation.

\subsection{Impact on the $D_{E}$ values}

\subsubsection{Comparison of the fitting functions}

Given that option A shows systematically the highest $S / N$ ratio, comparisons between the fitting functions were based on the $D_{\mathrm{E}}$ results derived from this option. Function Ti-2 and Ti-4 yield $D_{\mathrm{E}}$ values that are not significantly different at 1 sigma for 5/6 samples (Table 5). No apparent systematic deviation is observed. Four of these samples have a relative difference lower than $10 \%$, whereas for AB1104 this is of $16 \%$. The remaining sample (FN1001) shows a relative difference of $-21 \%$ between the $D_{\mathrm{E}}$ obtained with function Ti- 4 and Ti-2, but this is very likely explained by the large uncertainty on the ESR intensities measured (FN1001 is the sample showing the highest variability of the ESR intensities; Table 3). Function Ti- 5 yield $D_{\mathrm{E}}$ values that are systematically lower by -15 to $-32 \%$ in comparison with the results from Ti-2. Finally Ti-3 function provides $D_{\mathrm{E}}$ results that are very similar to those derived from $\mathrm{Ti}-2$ for four samples (relative difference $<10 \%$ ), but the six samples give $D_{\mathrm{E}}$ values that are in agreement at 1 sigma.
Adjusted $r^{2}$ values indicate that all functions overall show a good fitting (Table 6). On average function Ti-4 show the best fitting $\left(r^{2}=0.991\right)$, followed by Ti-2 $\left(r^{2}=0.989\right), \mathrm{Ti}-3\left(r^{2}=0.988\right)$ and then Ti-5 $\left(r^{2}=0.975\right)$. The latter is clearly limited by the non-linear trend of the decreasing domain of the curve (Fig. 7).

The magnitude of the $D_{\mathrm{E}}$ errors is also an indication about the reliability and precision of the fitting. In that regard, function $\mathrm{Ti}-2$ shows on average the lowest relative errors (8.0\%), followed by Ti-5 (11.9\%), Ti-4 (14.2\%) and then Ti-3 (24.2\%). The latter overall shows somewhat high errors for each of the fitted parameters (frequently $>100 \%$ ), indicating thus the function is very likely overparametrized. Indeed, $\mathrm{Ti}-3$ has the highest number of fitted parameters (6) among the five functions, which is clearly a problem given the limited number of points per DRC (12-14 depending on the sample). It is usually considered that $3-4$ points per fitted parameter (Lyons et al., 1992) are required for an optimum fitting, which means that a total of $18-24$ points should be included in the DRC when using the function Ti-3. Such an option may be hardly envisaged for ESR dating/dosimetry since it would imply very long 


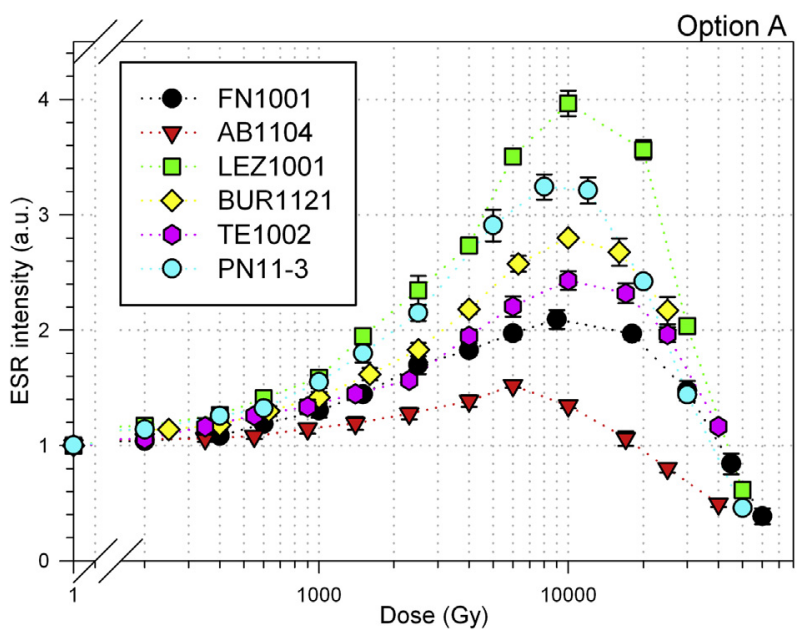

Fig. 5. Illustration of the variability of the radiation sensitivity between the samples for a given option (A).
Table 4

Relative mean ESR intensities derived from each option. For each sample, the ESR intensities were normalized to that obtained with option $\mathrm{A}$.

\begin{tabular}{llllll}
\hline & Option A & Option B & Option C & Option D & Option E \\
\hline BUR1121 & 1.00 & 0.87 & 0.34 & 0.67 & 0.33 \\
LEZ1001 & 1.00 & 0.88 & 0.27 & 0.64 & 0.36 \\
TE1002 & 1.00 & 0.94 & 0.35 & 0.68 & 0.32 \\
FN1001 & 1.00 & 0.89 & 0.19 & 0.65 & 0.35 \\
AB1104 & 1.00 & 0.94 & 0.37 & 0.66 & 0.34 \\
PN11-3 & 1.00 & 0.83 & 0.24 & 0.65 & 0.35 \\
Mean & 1.00 & 0.89 & 0.29 & 0.66 & 0.34 \\
\hline
\end{tabular}

ESR measurements at low temperature over several hours, and the stability of the spectrometer may become an issue.

Consequently, our results indicate that functions $\mathrm{Ti}-2$ and $\mathrm{Ti}-4$ may be considered as the best options: both functions yield very similar $D_{\mathrm{E}}$ values in most cases and have close goodness-of-fit (mean adj. $r^{2}=0.989$ and 0.991 ). However, the Ti-2 function has one less fitting parameter. This means that a DRC can be built with 12-16 experimental points, which is an advantage over the Ti- 4 for the present data set in terms of precision of the fitted parameters. The use of Ti- 4 would instead require ESR DRCs between 15 and 20 data points.

Table 3

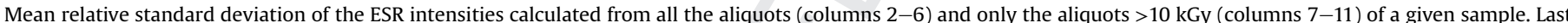
line: mean values calculated for each option.

\begin{tabular}{|c|c|c|c|c|c|c|c|c|c|c|}
\hline \multirow[t]{2}{*}{ Option } & \multicolumn{5}{|c|}{ All points } & \multicolumn{5}{|c|}{ Only points > $10 \mathrm{kGy}$} \\
\hline & A & B & $\mathrm{C}$ & D & $\mathrm{E}$ & A & B & C & $\mathrm{D}$ & $\mathrm{E}$ \\
\hline BUR1121 & $3.0 \%$ & $3.7 \%$ & $6.6 \%$ & $2.5 \%$ & $7.3 \%$ & $4.9 \%$ & $3.9 \%$ & $10.2 \%$ & $3.1 \%$ & $12.8 \%$ \\
\hline LEZ1001 & $3.3 \%$ & $4.7 \%$ & $13.0 \%$ & $3.6 \%$ & $7.0 \%$ & $3.8 \%$ & $4.7 \%$ & $20.5 \%$ & $4.0 \%$ & $7.2 \%$ \\
\hline TE1002 & $3.4 \%$ & $4.8 \%$ & $10.0 \%$ & $3.7 \%$ & $6.2 \%$ & $3.6 \%$ & $5.1 \%$ & $16.2 \%$ & $3.5 \%$ & $6.6 \%$ \\
\hline FN1001 & $4.3 \%$ & $8.5 \%$ & $32.1 \%$ & $6.0 \%$ & $11.8 \%$ & $9.3 \%$ & $12.3 \%$ & $51.9 \%$ & $10.0 \%$ & $27.0 \%$ \\
\hline AB1104 & $3.5 \%$ & $4.2 \%$ & $9.1 \%$ & $5.3 \%$ & $8.9 \%$ & $5.6 \%$ & $5.6 \%$ & $12.1 \%$ & $7.8 \%$ & $15.6 \%$ \\
\hline PN11-3 & $3.0 \%$ & $4.6 \%$ & $12.4 \%$ & $2.8 \%$ & $5.8 \%$ & $2.8 \%$ & $5.4 \%$ & $23.0 \%$ & $4.0 \%$ & $6.7 \%$ \\
\hline Mean & $3.4 \%$ & $5.1 \%$ & $13.9 \%$ & $4.0 \%$ & $7.8 \%$ & $5.0 \%$ & $6.2 \%$ & $22.3 \%$ & $5.4 \%$ & $12.6 \%$ \\
\hline
\end{tabular}

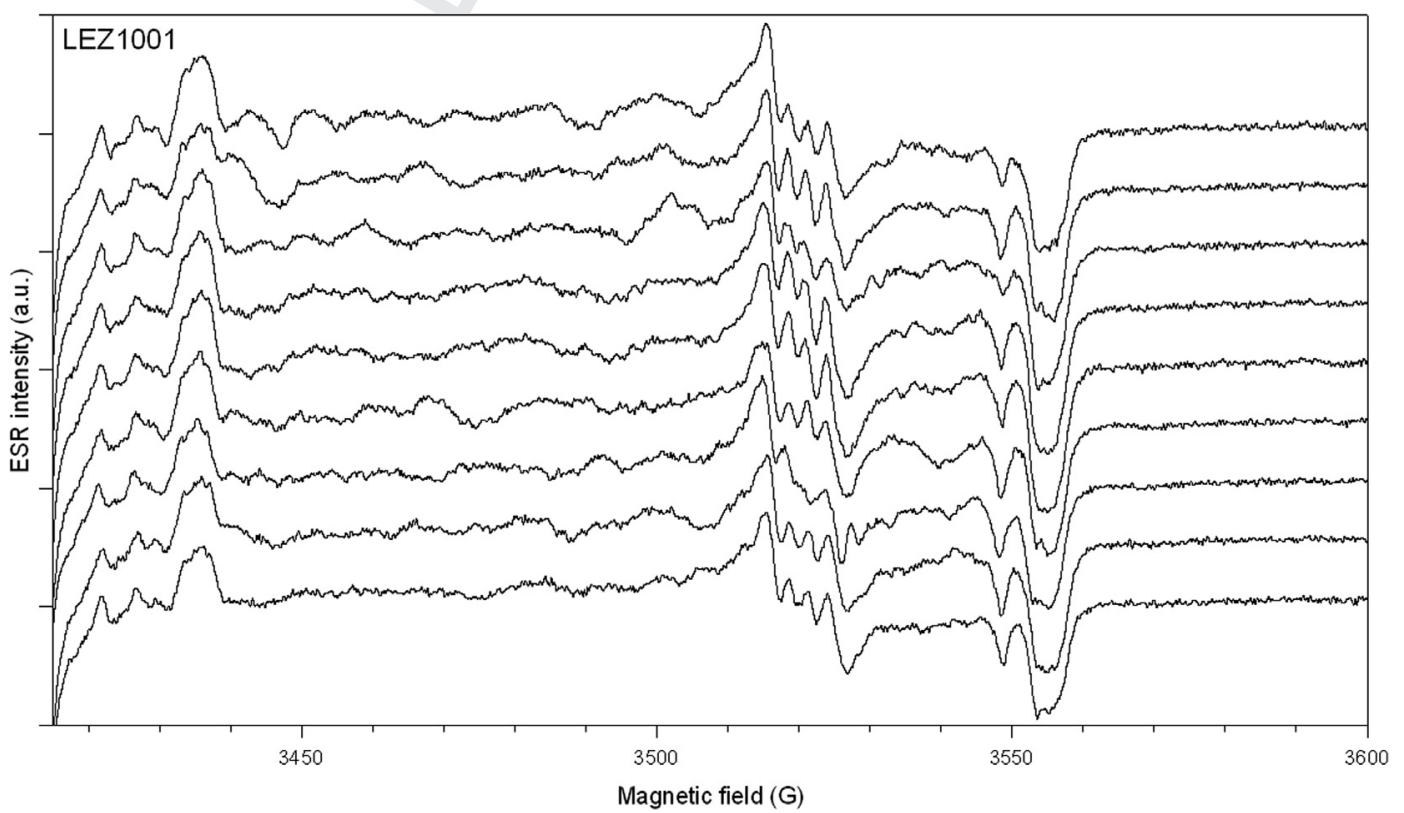

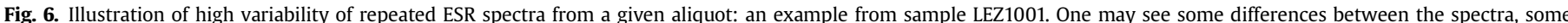

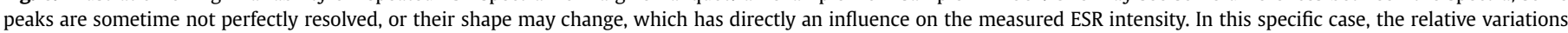
(1 s.d.) of the ESR intensity are of $5.4 \%$ for option A, $6.2 \%$ for option B, $14.7 \%$ for option C, $4.4 \%$ for option D, and $11.0 \%$ for option $\mathrm{E}$. 
Table 5

$D_{\mathrm{E}}$ values derived from the various fitting functions. Calculations were based on the ESR intensities derived from option A.

\begin{tabular}{llllll}
\hline & Ti-1 (SSE) & Ti-2 & Ti-3 & Ti-4 & Ti-5 \\
\hline BUR1121 & $1875 \pm 101$ & $2050 \pm 86$ & $2211 \pm 704$ & $2070 \pm 155$ & $1742 \pm 59$ \\
LEZ1001 & $1364 \pm 145$ & $1262 \pm 128$ & $1754 \pm 610$ & $1350 \pm 206$ & $1035 \pm 195$ \\
TE1002 & $2381 \pm 262$ & $2683 \pm 168$ & $2683 \pm 363$ & $2429 \pm 470$ & $2183 \pm 129$ \\
FN1001 & $1720 \pm 146$ & $2819 \pm 273$ & $2821 \pm 545$ & $2235 \pm 206$ & $1926 \pm 151$ \\
AB1104 & $2374 \pm 811$ & $2926 \pm 404$ & $2926 \pm 988$ & $3392 \pm 520$ & $2274 \pm 589$ \\
PN11-3 & $1158 \pm 135$ & $1361 \pm 56$ & $1669 \pm 199$ & $1366 \pm 65$ & $1030 \pm 239$ \\
\hline
\end{tabular}

\subsubsection{The SSE function}

Obviously, the SSE function can simply not describe the radiation bleaching process. Since this function has been systematically used in the past, the main question now lies on evaluating whether the use of the SSE induces any significant bias in the $D_{\mathrm{E}}$ evaluation. One of the main weaknesses of the SSE is that for some materials the maximum applied radiation dose $\left(D_{\max }\right)$ has a strong influence on the obtained $D_{\mathrm{E}}$ value: basically, the $D_{\mathrm{E}}$ value increases together with $D_{\text {max }}$. This correlation is usually used to highlight the inadequacy of a fitting model (e.g. Lyons, 1992; Grün, 1990). This phenomenon has been previously observed for fossil tooth enamel (Duval et al., 2009) and the Al center in quartz (Duval, 2012). In our case, because it is obviously impossible to fit the whole experimental data set with a SSE, we calculated the $D_{\mathrm{E}}$ values by considering the maximum ESR intensity as the last point of the DRC. Depending on the sample, this corresponds to a maximum given dose $\left(D_{\max }\right)$ of $9-12 \mathrm{kGy}$. $D_{\mathrm{E}}$ values are shown in Table 5 (column 2).

$D_{\mathrm{E}}$ values derived from the SSE are on average $14 \%$ smaller than those obtained from the function Ti-2. No apparent significant systematic deviation is observed, even if only one sample (LEZ1001) has higher $D_{\mathrm{E}}$ values with the SSE. The absolute relative difference is ranging between 8\% (LEZ1001) and 39\% (FN1001). For FN1001, the huge difference might be rather coming from the Ti-2 function, whose $D_{\mathrm{E}}$ value has previously found (section 4.4.1.) to be exceptionally higher than that derived from the Ti-4 (Table 5, Fig. 7). Consequently, this sample may be considered as a special case that should not be used for any further interpretation. When excluding FN1001 from the data set, the remaining $D_{\mathrm{E}}$ values derived from the SSE are on average $9 \%$ smaller than those from the Ti-2. Four samples are in agreement at 1 sigma, even AB1104, which shows a poor fitting as illustrated by the large relative $D_{\mathrm{E}}$ error (34\%) and the low $r^{2}$ value 0.879 (Table 6). The only exception is sample PN11-3.

Fig. 8 shows a plot of the evolution of the $D_{\mathrm{E}}$ value along the $D_{\max }$ in order to visualize the consistency of the results derived from both functions. $D_{\max }$ is ranging from 2.3 to $12 \mathrm{kGy}$ ( $n=$ number of aliquots; $n=7$ to $n=10$ ) for SSE since this function cannot describe the negative slope of the DRC at high doses. In contrast, Ti-2 requires this domain and was thus fitted on $D_{\max }$ ranging from 16 to $60 \mathrm{kGy}(n>10)$. The influence of $D_{\max }$ on the $D_{\mathrm{E}}$ value is usually considered as a good indicator of the correctness of the fitting function that is employed to describe the experimental data points. For the six samples, the $D_{\mathrm{E}}$ value calculated with Ti-2 function considering the entire data set $(n=13-14$ points, depending on the sample) is in close agreement with the $D_{\mathrm{E}}$ values obtained with the $D_{\max }=$ penultimate point $(n=12-13$ points, depending on the sample). In fact the variation in the $D_{\mathrm{E}}$ values is ranging from 1.2 to $6.7 \%$, i.e. systematically lower (except FN1001) than the variation observed with SSE (from 2.9 to $44.1 \%$ ). Consequently, the $D_{\mathrm{E}}$ value derived from Ti-2 fitted through the whole data set may be considered as the most reliable value and could be used as reference.
With SSE function, we hardly observe any systematic trend in the evolution of $D_{\mathrm{E}}$ together with $D_{\mathrm{max}}$, except, perhaps, that for five of the samples the $D_{\mathrm{E}}$ value apparently increases from $n=7$ to $n=8$ or 9 and then decreases to $n=10$. For $4 / 6$ samples, the $D_{\mathrm{E}}$ value derived from the SSE function when considering all the data points until the maximum ESR intensity $(n=10)$ is in agreement at one sigma with the reference $D_{\mathrm{E}}$ value. The exceptions are FN1001 and PN11-3. Nevertheless, the best agreement is never obtained with $n=10$ points but with $n=7\left(D_{\max }=2.3-2.5 \mathrm{kGy}\right)$ for 4 samples and with $n=9\left(D_{\max }=6.0-6.3 \mathrm{kGy}\right)$ for two samples (BUR1121, TE1002). These differences from one sample to another might be simply due to the experimental uncertainty on the ESR intensities. This suggests that the first increasing domain of the DRC very likely follows a SSE behavior at least up to 6.0-6.5 kGy, while this behavior starts changing at higher doses.

To conclude, it seems that the SSE function yield $D_{\mathrm{E}}$ values that are in relative agreement with those derived from the Ti- 2 in most of the cases. Nevertheless, when using only the SSE, there is no way to control the reliability of the $D_{\mathrm{E}}$ value that is obtained since it is dependent on the accuracy of the ESR intensities and saturation cannot be reached. In addition, one may reasonably question the reason to keep working with a function that is known to be incorrect.

\subsubsection{Comparison of the $D_{E}$ derived from the various options to evaluate the ESR intensities}

Tables 7 and 8 show the numerical fitting results obtained with function Ti-2 for all the samples. Options A and D yield similar $D_{\mathrm{E}}$ estimates. On six samples, four of them (BUR1121, LEZ1001, TE1002 and PN11-3) show a relative $D_{\mathrm{E}}$ difference $<5 \%$, and no apparent bias is observed. The two other samples (FN1001, AB1104) show a somewhat higher value, between 10 and $20 \%$ of relative difference between each option, but the $D_{\mathrm{E}}$ values nevertheless overlap at 1 sigma (Table 7). This larger difference, may be very likely explained by the fitting which is not as good as for the three other samples, as indicated by the $r^{2}$ value $<0.98$ and the magnitude of the $D_{\mathrm{E}}$ errors (>13\%) for one of the two options (Option D for FN1001 and option A for AB1104, Tables 7 and 8). Therefore, these results indicate that under normal conditions both options yield a quite similar $D_{\mathrm{E}}$ value, suggesting that the previously identified interferences (i.e., the $\mathrm{Al}$ signal for option A and the Ti-H line at $g=1.915$ for option $\mathrm{D}$ ) have a negligible impact on the $D_{\mathrm{E}}$ results. These results are in good agreement with those obtained by Tissoux et al. (2007). Part of the differences in the $D_{\mathrm{E}}$ values is very likely due to the experimental uncertainty associated to the ESR intensities.

Four out of six samples (BUR1121, LEZ1001, FN1001, PN11-3) show $D_{\mathrm{E}}$ values within $<10 \%$ between options $A$ and E. Five samples have $D_{\mathrm{E}}$ values within \pm 1 sigma, the exception being sample TE1002. Nevertheless, for one of them (AB1104), the fitting is obviously not satisfactory, given the very low $r^{2}$ value $<0.87$ and the resulting very high $D_{\mathrm{E}}$ error $(3552 \pm 1316 \mathrm{~Gy})$. Similar observations may be done when comparing option $\mathrm{E}$ with $\mathrm{D}$. Such results suggest that the supposedly interference of the $\mathrm{Ti}-\mathrm{H}$ line in options A and D previously mentioned in Section 4.1 has finally in overall very little influence. Consequently, one may consider that these options are mostly dominated by the Ti-Li signal. Nevertheless, if option E may potentially be a good alternative to options A and D for $D_{\mathrm{E}}$ evaluation, it shows a clear disadvantage in terms of magnitude of the ESR intensity, on average only $34 \%$ of the ESR intensity of the option A (Table 4). This simply leads to a much smaller $S / N$ ratio and thus less repeatable measurements, as shown by the magnitude of the average relative standard deviation of the ESR intensity that are ranging from $6.2 \%$ to $11.8 \%$ depending on the sample (Table 3). Goodness-of-fit is also not as good as with the other options ( $r^{2}=0.966$ in average). 
Table 6

Adjusted $r^{2}$ values derived from the various fitting functions. Calculations were based on the ESR intensities derived from option A.

\begin{tabular}{llllll}
\hline & Ti-1 $($ SSE $)$ & Ti-2 & Ti-3 & Ti-4 & Ti-5 \\
\hline BUR1121 & 0.998 & 0.998 & 0.998 & 0.998 & 0.998 \\
LEZ1001 & 0.994 & 0.983 & 0.990 & 0.985 & 0.964 \\
TE1002 & 0.991 & 0.993 & 0.991 & 0.991 & 0.992 \\
FN1001 & 0.994 & 0.984 & 0.979 & 0.992 & 0.992 \\
AB1104 & 0.879 & 0.978 & 0.970 & 0.982 & 0.940 \\
PN11-3 & 0.993 & 0.998 & 0.999 & 0.999 & 0.964 \\
Mean & 0.975 & 0.989 & 0.988 & 0.991 & 0.975 \\
\hline
\end{tabular}

Consequently, our results indicate that the best ways to evaluate the signal for Ti-Li center are options A and D: they show the best compromise between $S / N$ ratio, measurement repeatability and
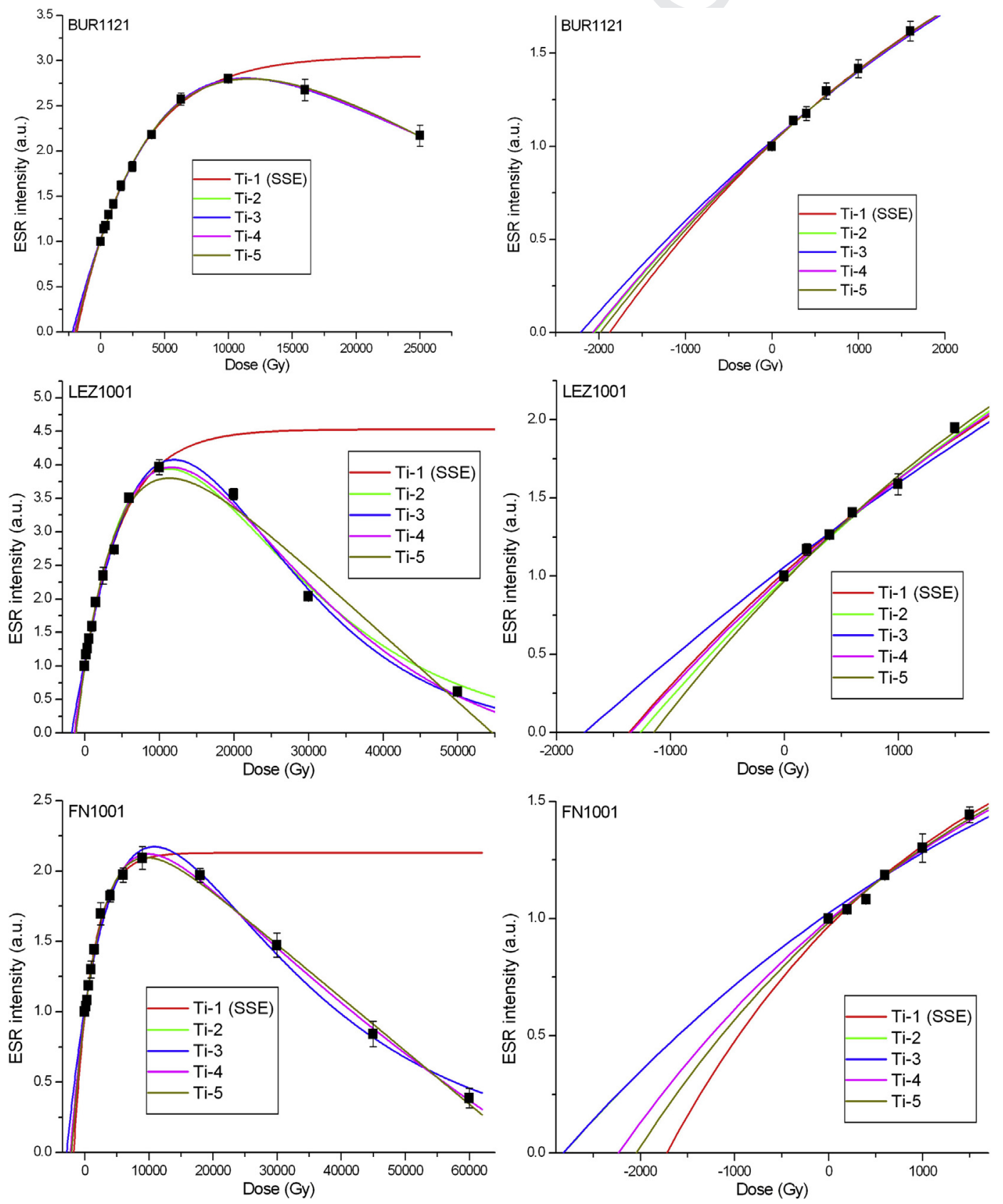

Fig. 7. Various functions fitted through the experimental data points (Option A) obtained for 3 samples (BUR1121, LEZ1001 and FN1001). goodness-of-fit and should be both systematically extracted for each sample. A cross check of the $D_{\mathrm{E}}$ obtained with the 2 options may help to get some quality control on the results. In addition, the measurement of option $\mathrm{E}$ could be a way to assess the relative weight of the $\mathrm{Ti}-\mathrm{H}$ line on the $D_{\mathrm{E}}$ value derived from option $\mathrm{A}$ and $\mathrm{E}$.

Option B shows $D_{\mathrm{E}}$ values that are lower than those obtained with option A for four of the samples (Table 7). The exceptions are LEZ1001 and PN11-3, but the difference is not significant for the latter. We do not have any explanations about why LEZ1001 lies outside of this pattern since both options do show an apparent good fitting. Nevertheless, the four other samples have $D_{\mathrm{E}}$ values that are between 9 and 25\% lower than those derived from the option $\mathrm{A}$ and more than 2 times higher than results obtained with option C. This pattern is not surprising, since option B is a mix of 

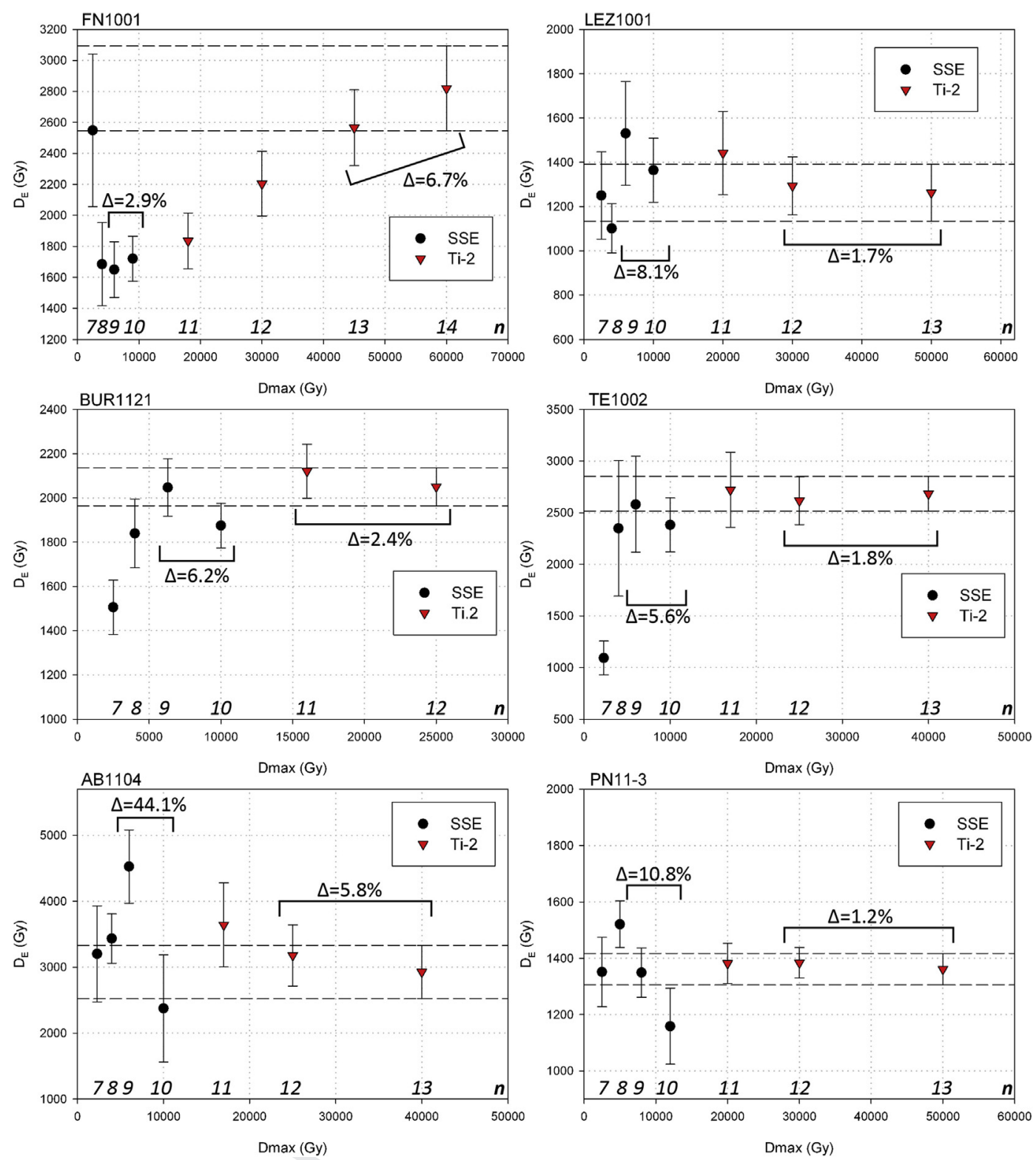

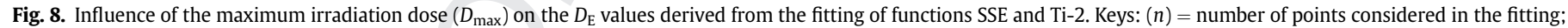
$\Delta=$ relative standard deviation (in \%).

contributions from $\mathrm{Ti}-\mathrm{Li}$ and $\mathrm{Ti}-\mathrm{H}$ lines. Overall, the goodness of fit for option B is lower than that of option A and D (Table 8).

Option $\mathrm{C}$ is the only way to measure the $\mathrm{Ti}-\mathrm{H}$ signal alone. The $D_{\mathrm{E}}$ values are systematically and significantly smaller than those obtained for the option $A$, giving values for option $C$ ranging from 0.26 to $0.86^{*} D_{\mathrm{E}}$ (option A). Overall, the fitting is systematically not good for each of the sample, since the $r^{2}$ range from 0.85 to 0.97 , which lead to reasonably question the reliability of the $D_{\mathrm{E}}$ values that are calculated. It is worth noting that fitting is not better with the functions other than Ti-2. Option C is the option that shows the lowest signal intensity of the 5 options, about $29 \%$ on average of the absolute ESR intensity of the option A (Table 4). This clearly has an impact on the associated mean relative standard deviation, from 6 to $32 \%$ when considering all points, and from 10 to $52 \%$ for the points $>10 \mathrm{kGy}$ (Table 3 ). This underlines the large uncertainty associated to the ESR intensities, and the actual data set does not allow to get any reliable interpretation for option $\mathrm{C}$. It seems that a specific work will have to be performed on significantly improving the $S / N$ ratio of that signal in order to draw any convincing conclusion.

\subsection{Summary}

The main findings derived from our results may be summarized as follows:

- The ESR signal is characterized by a quite low ESR intensity which may be 10 times lower than that of the Al center. Consequently, the main issue consists in optimizing the $S / N$ ratio in order to get meaningful results. This can be done by optimizing several factors of the experimental conditions, such as the sample mass in the tube, the acquisition parameters (e.g. microwave power, number of scans, conversion time, time constant, etc.) and the number of repeated measurements with 
Table 7

$D_{\mathrm{E}}$ values calculated from the various options used to evaluate the ESR intensity. Calculations were performed using the Ti-2 fitting function.

\begin{tabular}{lllrll}
\hline & Option A & Option B & \multicolumn{1}{c}{ Option C } & Option D & Option E \\
\hline BUR1121 & $2050 \pm 86$ & $1857 \pm 216$ & $730 \pm 133$ & $1984 \pm 142$ & $1996 \pm 194$ \\
LEZ1001 & $1262 \pm 128$ & $1611 \pm 234$ & $1081 \pm 370$ & $1314 \pm 145$ & $1177 \pm 202$ \\
TE1002 & $2683 \pm 168$ & $2002 \pm 321$ & $850 \pm 321$ & $2724 \pm 274$ & $2255 \pm 235$ \\
FN1001 & $2819 \pm 273$ & $2289 \pm 250$ & $725 \pm 310$ & $2513 \pm 453$ & $3042 \pm 223$ \\
AB1104 & $2926 \pm 404$ & $2298 \pm 381$ & $1146 \pm 245$ & $2435 \pm 155$ & $3552 \pm 1316$ \\
PN11-3 & $1361 \pm 56$ & $1402 \pm 138$ & $730 \pm 144$ & $1353 \pm 59$ & $1310 \pm 76$ \\
\hline
\end{tabular}

Table 8

Adjusted $r^{2}$ values derived from the fitting of Ti-2 function for each option.

\begin{tabular}{llllll}
\hline & Option A & Option B & Option C & Option D & Option E \\
\hline BUR1121 & 0.998 & 0.986 & 0.958 & 0.996 & 0.991 \\
LEZ1001 & 0.983 & 0.971 & 0.903 & 0.987 & 0.971 \\
TE1002 & 0.993 & 0.960 & 0.852 & 0.982 & 0.983 \\
FN1001 & 0.984 & 0.980 & 0.898 & 0.949 & 0.990 \\
AB1104 & 0.978 & 0.958 & 0.949 & 0.995 & 0.862 \\
PN11-3 & 0.998 & 0.990 & 0.971 & 0.998 & 0.997 \\
Mean & 0.989 & 0.974 & 0.922 & 0.984 & 0.966 \\
\hline
\end{tabular}

or without tube rotations in the cavity. However, this leads to measurements that are significantly longer than those required for the Al center: in our work, the measurement of all the aliquots of a given sample requires between 2.5 and $3 \mathrm{~h}$ of measurements, while it takes $\sim 1 \mathrm{~h}$ per sample for the Al center (Duval and Guilarte Moreno, 2012). Even with those conditions, the $S / N$ ratio was very low, sometimes $<2$, which indicate that the reliability of the results obtained for some options may be reasonable questioned (e.g. options $C$ and E). Nevertheless, our results show the necessity to carry out repeated ESR measurements of the Ti-center, in order to get an accurate mean ESR intensity.

- Options A and D may be used for the evaluation of the intensity of the Ti-Li center, since the ESR signals have the highest $S / N$ ratio and the ESR measurements show the best precision. The two options yield consistent $D_{\mathrm{E}}$ values at $1 \sigma$ for the six samples. We recommend the measurement of both options to cross check the reliability of the $D_{\mathrm{E}}$ results.

- Option E was a priori the only way to measure the Ti-Li center alone, unlike options $\mathrm{A}$ and $\mathrm{D}$ that were apparently made by a mixture of $\mathrm{Ti}-\mathrm{Li}$ and $\mathrm{Ti}-\mathrm{H}$. However, $D_{\mathrm{E}}$ values derived from the three options are in overall in relative good agreement for five out of six samples, suggesting thus that the influence of the $\mathrm{Ti}-\mathrm{H}$ line in options A and D may be neglected in most cases. For this reason, we recommend the measurement of option $E$ for comparison, i.e. a way to check the weight of the $\mathrm{Ti}-\mathrm{H}$ line in the $D_{\mathrm{E}}$ value that is obtained with options A and D. In contrast, the use of the option $\mathrm{E}$ alone for dosimetric purpose has some disadvantages, since ESR measurements are not as repeatable as those for the options $A$ and $D$, resulting in a significantly lower goodness-of-fit and thus higher $D_{\mathrm{E}}$ errors.

- Option $\mathrm{B}$ is a mixture of $\mathrm{Ti}-\mathrm{Li}$ and $\mathrm{Ti}-\mathrm{H}$ centers which might be of special interest when working on a combination of both signals (Beerten and Stesmans, 2006). However, the poor goodness of fit obtained with $\mathrm{Ti}-2$ indicates that this function does not exactly describe the behavior of these samples, and neither do the other functions. Further work is needed in that direction.

- The $\mathrm{Ti}-\mathrm{H}$ signal at $\mathrm{g}=1.915$ (option $\mathrm{C}$ ) shows obviously a different behavior to the other signals that have been measured. This signal saturates at lower doses and provides $D_{\mathrm{E}}$ values that are systematically the lowest of the data set (Table 6). However, it would result hazardous to draw any definitive conclusions and interpretation based on this signal, given the very low $S / N$ ratio, the resulting low precision of the ESR intensities and the poor goodness-of-fit that are obtained with function Ti-2. To get meaningful information, a significant effort will have to be done on optimizing the experimental conditions for the specific measurement of this center, since it potentially offers some interesting characteristics for dating purpose such as higher radiation sensitivity and faster bleaching kinetics (e.g. Tissoux et al., 2007) than other centers.

- Function Ti-2 should be used to fit the DRC of the Ti-Li center, since it systematically shows a good fitting and has a relative small number of parameters (4) to fit. Nevertheless the use of this function requires: (i) a DRC with 12-16 experimental points, (ii) irradiation doses $>10 \mathrm{kGy}$ in order to get the radiation bleaching process, with at least 3-4 points, up to doses of $40 \mathrm{kGy}$. Otherwise, the fitting becomes complicated and the accuracy of the results may be reasonably questioned. The function Ti- 4 may be used in parallel to cross check the reliability of the $D_{\mathrm{E}}$ values.

- The SSE function apparently provides $D_{\mathrm{E}}$ results that are somewhat lower than those derived from the function Ti-2, but remain overall relatively consistent at $1 \sigma$. This suggests that the increasing domain of the DRC might be described by this function, at least up to 6.0-6.5 kGy. However, the reliability of the $D_{\mathrm{E}}$ values seems to be highly dependent on the accuracy of the ESR intensities that are measured. In addition, there is no apparent reason to keep using the SSE function since it does simply not describe the behavior of the Ti signal at high doses (>10 kGy).

- Our results highlight the variability that may exist between the samples. Depending on the parameters that are studied (evaluation of the ESR intensity, repeatability of the measurements, fitting function), some trends may be identified, but at the same time, there are systematically a few samples that may behave differently from the others, simply because these are experimental data. It is thus crucial to start defining some criteria to evaluate the quality of the data and the reliability of the results that are obtained. In that regard a few recommendations may be done. For the ESR intensity evaluation of the $\mathrm{Ti}-\mathrm{Li}$ center, options A, D and E should be extracted. The fitting process evaluation could be done with both function Ti-2 and Ti-4. All results should be cross-checked, and if all $D_{\mathrm{E}}$ values are within error, this may be a good indicator of the reliability of the results. In addition, Tables 6 and 8 show that the goodness-of-fit associated to the DRCs derived from the signal Ti-center is overall lower than that afforded for signals from other materials: the adjusted $r^{2}$ values are mostly somewhere between 0.98 and 0.99 , whereas they usually are $>0.99$ for DRCs of tooth enamel (Duval et al., 2013) or with the Al center in quartz (Duval, 2012). Because a function fitted through highly scattered data points may result in a meaningless $D_{\mathrm{E}}$ value, we would suggest the development of empirical criteria for defining a good fitting with Origin software series. To get a reliable $D_{\mathrm{E}}$ value from the mathematical point of view, it important to have an acceptable goodness-of-fit and to make sure that the function is not overparametrized in comparison with the data set available (see Duval et al., 2013; Lyons et al., 1992). Based on our experience with the Ti center we would recommend to make sure that (i) $r^{2}>0.98$ and (ii) relative errors on the fitted parameters are $<50 \%$. If fitting results do not fulfill these two criteria, then their reliability may be reasonably questioned, since it means that the fitting function does not fully describe the experimental data set. This is usually either because the choice of the function is wrong or because the ESR intensities are not accurate. 


\section{Conclusion}

The present work highlights some of the specificities of the $\mathrm{Ti}$ center and contributes to refine its potential for dosimetric/dating purpose by studying several sources of uncertainty such as the evaluation of the ESR intensity, the precision of the measurements and the fitting functions. Nevertheless, it has to be completed in the future by the study of other specific points, such as the potential of peak deconvolution for the ESR intensity evaluation, which may possibly be less dependent to high frequency variability of the ESR intensity than the classic peak-to-peak or peak-to-baseline measurements, or the impact of data weighting during the fitting on the final $D_{\mathrm{E}}$ values.

Finally, it is worth reminding that even if the Ti-2 function is obviously more appropriate than the SSE to fit experimental data points of the Ti center, this does not necessarily mean that the derived $D_{\mathrm{E}}$ values are correct. For example, the build-up of the natural ESR signal in the geological past may have been different to that reconstructed from additive dose points. Additional experiments focused on the comparison of the additive dose and regenerative dose method will have to be carried out in that regard. In addition, previous work highlighted the systematic $D_{\mathrm{E}}$ overestimation and underestimation provided by the $\mathrm{Ti}-\mathrm{Li}$ and $\mathrm{Ti}-\mathrm{H}$ centers, respectively (Beerten and Stesmans, 2006; Tissoux et al., 2007, 2008). Beerten and Stesmans (2006) also suggested that a total $\mathrm{Ti}$ signal derived from the sum of the $\mathrm{Ti}-\mathrm{Li}$ and $\mathrm{Ti}-\mathrm{H}$ signals may lead to correct $D_{\mathrm{E}}$ results. Other authors have used the apparent consistency between the $D_{\mathrm{E}}$ values derived from Al center and $\mathrm{Ti}-\mathrm{Li}$ centers as a criterion to check the validity of the age results (Rink et al., 2007; Burdette et al., 2012). However, the reliability of the interpretations derived from all these results is mainly limited by the fact that SSE functions were systematically used for both $\mathrm{Al}$ and Ti-Li centers, whereas several studies (e.g. Duval, 2012) including the present one, have showed that each center has a specific radiation behavior that is quite different to the SSE. Therefore, it is more crucial than ever to develop future ESR dating studies on precisely known-age $/ D_{\mathrm{E}}$ samples in order to better define the exact potential and limits of the Ti-center for dosimetric/ dating purpose.

\section{Uncited references}

Ikeya, 1993, Sahnouni et al., 2011, Toyoda et al., 1995, Toyoda and Ikeya, 1991.

\section{Acknowledgments}

This study was partially sponsored by the projects CGL201016821 and CEN001B10-2 from the Spanish Ministry of Science and Innovation and the Junta de Castilla y León, respectively. The authors thank their colleagues, archaeologists and geologists, for providing access to the sampling sites. Data evaluation and manuscript redaction were mainly carried out during MD's 3 month stay at the Research School of Earth Sciences, the Australian National University, which was funded by a José Castillejo Mobility Fellowship CAS12/00251 from the Spanish Ministry for Education, Culture and Sport. We thank the two anonymous reviewers for helpful comments.

\section{References}

Arzarello, M., Pavia, G., Peretto, C., Petronio, C., Sardella, R., 2012. Evidence of an Early Pleistocene hominin presence at Pirro Nord (Apricena, Foggia, southern Italy): P13 site. Quat. Int. 267, 56-61.
Beerten, K., Stesmans, A., 2006. Some properties of Ti-related paramagnetic centres relevant for electron spin resonance dating of single sedimentary quartz grains. Appl. Radiat. Isotopes 64, 594-602.

Burdette, K.E., Rink, W.J., Mallinson, D.J., Means, G.H., Parham, P.R., 2012. Electron spin resonance optical dating of marine, estuarine, and aeolian sediments in Florida, USA. Quat. Res. 79 (1), 66-74.

Carbonell, E., Bermudez de Castro, J., Parés, J., Pérez-González, A., Cuenca-Bescos, G., Olle, A., Mosquera, M., Huguet, R., Made, J. v. d, Rosas, A., Sala, R., Vallverdu, J., Garcia, N., Granger, D., Martinon-Torres, M., Rodriguez, X., Stock, G., Verges, J., Allue, E., Burjachs, F., Caceres, I., Canals, A., Benito, A., Diez, C., Lozano, M., Mateos, A., Navazo, M., Rodriguez, J., Rosell, J., Arsuaga, J., 2008. The first hominin of Europe. Nature 452, 465-470.

Charalambous, S., Petridou, C., 1976. The thermoluminescence behavior of LiF(TLD100 ) for doses up to 100 mrad. Nucl. Instrum. Methods 137, 441-444.

Crochet, J.-Y., Welcomme, J.-L., Ivorra, J., Ruffet, G., Boulbes, N., Capdevila, R., Claude, J., Firmat, C., Métais, G., Michaux, J., Pickford, M., 2009. Une nouvelle faune de vertébrés continentaux, associée à des artefacts dans le Pléistocène inférieur de l'Hérault (Sud de la France), vers 1,57 Ma. C. R. Palevol. 8, 725-736.

Duttine, M., Villeneuve, G., Bechtel, F., Demazeau, G., 2002. Caractérisation par résonance paramagnétique électronique (RPE) de quartz naturels issus de différentes sources. C. R. Geosci. 334 (13), 949-955.

Duttine, M., 2005. Recherche de provenance de quartz et d'obsidiennes préhistoriques en Europe occidentale - Apports de la résonance paramagnétique électronique (RPE) (PhD thesis). University of Bordeaux 3.

Duval, M., 2012. Dose response curve of the ESR signal of Aluminum center in quartz grains extracted from sediment. Anc. TL 30 (2), 41-49.

Duval, M., Guilarte Moreno, V., 2012. Assessing the influence of the cavity temperature on the ESR signal of aluminum center in quartz grains extracted from sediment. Anc. TL 30 (2), 51-56.

Duval, M., Grün, R., Falguères, C., Bahain, J.-J., Dolo, J.-M., 2009. ESR dating of Lower Pleistocene fossil teeth: limits of the single saturating exponential (SSE) model for the equivalent dose determination. Radiat. Meas. 44, 477-482.

Duval, M., Guilarte Moreno, V., Grün, R., 2013. ESR dosimetry of fossil enamel: some comments about measurement precision, long-term signal fading and dose-response curve fitting. Radiat. Prot. Dosim. 157 (4), 463-476.

Euler, F.K., Kahan, A., 1987. Radiation effects and anelastic loss in germanium-doped quartz. Phys. Rev. B 35 (9), 4351-4359.

Götze, J., Plötze, M., Habermann, D., 2001. Origin, spectral characteristics and practical applications of the cathodoluminescence $(\mathrm{CL})$ of quartz - a review. Mineralogy Petrology 71, 225-250.

Grün, R., 1990. Dose response of the paramagnetic centre at $g=2.0007$ in Corals. Anc. TL 8, 20-22.

Grün, R., Brumby, S., 1994. The assessment of errors in past radiation doses extrapolated from ESR/TL dose-response data. Radiat. Meas. 23, 307-315.

Hayes, R.B., Haskell, E.H., Kenner, G.H., 1998. An assessment of the LevenbergMarquardt fitting algorithm on saturating exponential data sets. Anc. TL 16 (2), 57-62.

Ikeya, M., 1993. In: Zimmerman, M., Whitehead, N. (Eds.), New Applications of Electron Spin Resonance - Dating, Dosimetry and Microscopy. World Scientific Publishing.

Lawless, J.L., Chen, R., Lo, D., Pagonis, V., 2005. A model for non-monotonic dose dependence of thermoluminescence (TL). J. Phys. Condens. Matter 17, 737-753.

Lemartinel, B., 1997. Plan miocène et épandages siliceux (cf. rañas) au pied des Monts Ibériques Occidentaux/Miocene planation surface and siliceous deposits on the piedmonts of the Western Iberian Ranges. Géomorphol. Relief Process. Environ. 3 (3), 195-207.

Liu, C.-R., Yin, G.M., Gao, L., Bahain, J.-J., Li, J.-P., Lin, M., Chen, S.-M., 2010. ESR dating of Pleistocene archaeological localities of the Nihewan Basin, North China preliminary results. Quat. Geochronol. 5, 385-390.

Liu, C.-R., Yin, G.-M., Fang, F., Voinchet, P., Deng, C.-L., Han, F., Li, J.-P., Song, W.-J., Wang, D., Bahain, J.-J., 2013. ESR dating of the Donggutuo Palaeolithic site in the Nihewan Basin, northern China. Geochronometria 40 (4), 348-354.

Lyons, R.G., Brennan, B.J., Hosking, P.L., 1992. Estimation of accumulated dose and its uncertainties: potential pitfalls in curve fitting. Anc. TL 10, 42-49.

Maghraby, A., 2010. Uncertainty attributed to signal averaging in a single averaged alanine EPR spectrum for low-dose applications. Radiat. Prot. Dosim. 143 (1), $12-16$.

Martínez-Navarro, B., Turq, A., Agusti Ballester, J., Oms, O., 1997. FuenteNueva-3 (Orce, Granada, Spain) and the first human occupation of Europe. J. Hum. Evol. 33, 611-620.

Okada, M., Rinneberg, H., Weil, J.A., Wright, P.M., 1971. EPR of $\mathrm{Ti}^{3+}$ centers in aquartz. Chem. Phys. Lett. 11, 275-276.

Oms, O., Anadón, P., Agustí, J., Julià, R., 2011. Geology and chronology of the continental Pleistocene archeological and paleontological sites of the Orce area (Baza basin, Spain). Quat. Int. 243 (1), 33-43.

Pavia, M., Zunino, M., Coltorti, M., Angelone, C., Arzarello, M., Bagnus, C., Bellucci, L., Colombero, S., Marcolini, F., Peretto, C., Petronio, C., Petrucci, M., Pieruccini, P., Sardella, R., Tema, E., Villier, B., Pavia, G., 2012. Stratigraphical and palaeontological data from the Early Pleistocene Pirro 10 site of Pirro Nord (Puglia, south eastern Italy). Quat. Int. 267, 40-55.

Rink, W.J., Bartoll, J., Schwarcz, H.P., Shane, P., Bar-Yosef, O., 2007. Testing the reliability of ESR dating of optically exposed buried quartz sediments. Radiat. Meas. 42, 1618-1626.

Rinneberg, H., Weil, J.A., 1972. EPR studies of $\mathrm{Ti}^{3+}-\mathrm{H}^{+}$centers in X-irradiated alpha quartz. J. Chem. Phys. 56, 2019-2028. 
Sahnouni, M., de Heinzelin, J., 1998. The site of Ain Hanech Revisited: new investigations at this Lower Pleistocene site in Northern Algeria. J. Archaeol. Sci. 25 (11), 1083-1101.

Sahnouni, M., Van der Made, J., Everett, M., 2011. Ecological background to PlioPleistocene hominin occupation in North Africa: the vertebrate faunas from Ain Boucherit, Ain Hanech and El-Kherba, and paleosol stable-carbon-isotope studies from El-Kherba, Algeria. Quat. Sci. Rev. 30 (11-12), 1303-1317.

Shimada, A., Takada, M., Toyoda, S., 2013. Characteristics of ESR signals and TLCLs of quartz included in various source rocks and sediments in japan: a clue to sediment provenance. Geochronometria. http://dx.doi.org/10.2478/s13386-013-0111-z.

Toyoda, S., Goff, F., Ikeda, S., Ikeya, M., 1995. ESR dating of quartz phenocrysts in the El Cajete and Battleship Rock Members of Valles Rhyolite, Valles Caldera, New Mexico. J. Volcanol. Geotherm. Res. 67, 29-40.

Toyoda, S., Ikeya, M., 1991. Thermal stabilities of paramagnetic defects and impurity centers in quartz: basis for ESR dating of thermal history. Geochem. J. 25, 437-445.

Toyoda, S., Voinchet, P., Falguères, C., Dolo, J.-M., Laurent, M., 2000. Bleaching of ESR signals by the sunlight: a laboratory experiment for establishing the ESR dating of sediments. Appl. Radiat. Isotopes 52, 1357-1362.

Tissoux, H., Falguères, C., Voinchet, P., Toyoda, S., Bahain, J.-J., Despriée, J., 2007. Potential use of Ti-center in ESR dating of Fluvial Sediment. Quat. Geochronol. 2 $(1-4), 367-372$.
Tissoux, H., Toyoda, S., Falguères, C., Voinchet, P., Takada, M., Bahain, J.J., Despriée, J. 2008. ESR dating of quartz from two different Pleistocene deposits using $\mathrm{Al}$ and Ti-centers. Geochronometria 30, 23-31.

Tissoux, H., Voinchet, P., Lacquement, F., Prognon, F., Moreno, D., Falguères, C. Bahain, J.-J., Toyoda, S., 2012. Investigation on non-optically bleachable components of ESR Aluminium signal in quartz. Radiat. Meas. 47, 894-899.

Voinchet, P., Falguères, C., Laurent, M., Toyoda, S., Bahain, J.-J., Dolo, J.-M., 2003. Artificial optical bleaching of the aluminium center in quartz - implications to ESR dating of sediments. Quat. Sci. Rev. 22 (10-13), 1335-1338.

Walther, R., Zilles, D., 1994. ESR studies on bleached sedimentary quartz. Quat. Sci. Rev. Quat. Geochronol. 13, 611-614.

Weil, J.A., 1984. A review of electron spin spectroscopy and its application to the study of paramagnetic defects in crystalline quartz. Phys. Chem. Miner. 10 149-165.

Woda, C., Wagner, G.A., 2007. Non-monotonic dose dependence of the Ge- and Ticentres in quartz. Radiat. Meas. 42, 1441-1452.

Zhao, J., Lai, Z., Liu, S., Song, Y., Li, Z., Yin, X., 2012. OSL and ESR dating of glacial deposits and its implications for glacial landform evolution in the Bogeda Peak area, Tianshan range, China. Quat. Geochronol. 10, 237-243. 